\title{
Pushing towards ehealth for iraqi hypertensives: an integrated class association rules into SECI model
}

\author{
Ahmed Aljuboori ${ }^{1}$, Lubab Ahmed Tawfeeq ${ }^{2}$, Khamis A. Al-Karawi ${ }^{3}$ \\ ${ }^{1,2}$ Department of Computer Science, College of Education for Pure Science Ibn-Al-Haitham/University of Baghdad, Iraq \\ ${ }^{3}$ Diyala University, Iraq
}

\begin{tabular}{|c|c|}
\hline Article Info & ABSTRACT \\
\hline $\begin{array}{l}\text { Article history: } \\
\text { Received Nov 15, } 2020 \\
\text { Revised Jan } 17,2021 \\
\text { Accepted Jan 30, } 2021\end{array}$ & $\begin{array}{l}\text { This paper highlights the barriers that have led to a delay in the } \\
\text { implementation of E-health services in Iraq. A new framework is proposed to } \\
\text { improve the E-health sector using a SECI model which describes how } \\
\text { explicit and tacit knowledge is generated, transferred, and recreated in } \\
\text { organizations through main stages (socialization, externalization, } \\
\text { combination and internalization). Class association rules (CARs) is }\end{array}$ \\
\hline $\begin{array}{l}\text { Keywords: } \\
\text { Class association rules } \\
\text { Hypertension } \\
\text { Iraqi health services } \\
\text { Knowledge management } \\
\text { SECI model }\end{array}$ & $\begin{array}{l}\text { Integrated to mine the SECI model by extracting related rules which } \\
\text { correspond to the medical advice. The proposed framework (SECICAR) can } \\
\text { be done through a web portal to assemble healthcare professionals, patients } \\
\text { in one environment. SECICAR will be applied to the hypertension } \\
\text { community to show that disease if left untreated, frequently leads to serious } \\
\text { illnesses such as heart disease. The SECICAR aims to facilitate the } \\
\text { dissemination of tacit knowledge, which is explicit to hypertensives, in the } \\
\text { form of strategies, guidelines and best practices. The validation of the } \\
\text { SECICAR results displays satisfactory accuracy and reliability. Heuristic } \\
\text { evaluation was used to test the web portal, the participants stating that there } \\
\text { were no major issues regarding its usability. }\end{array}$ \\
\hline
\end{tabular}

This is an open access article under the CC BY-SA license.

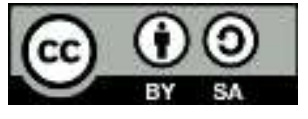

\section{Corresponding Author:}

Ahmed Aljuboori

Department of Computer Science

College of Education for Pure Science Ibn-Al-Haitham/University of Baghdad

Antara Bin Shaddad Square, Baghdad, Iraq

Email: a.s.aljuboori@ihcoedu.uobaghdad.iq.edu

\section{INTRODUCTION}

As a third world country, Iraq still suffers from the insufficient implementation of a national health system (NHS). Just as E-health plays a major role in the enhancement of healthcare services in many countries, Iraq also aims to implement different e-health solutions to improve its healthcare services. Nevertheless, there are significant barriers that have been recognized as impacting the successful implementation of different e-health solutions. In addition, Iraq along with many other countries suffers from a high frequency of hypertension [1]. Along with health difficulties associated with chronic diseases, hypertension exacerbates the complications that are linked to poor or unhealthy lifestyles and the use of medications. Moreover, although self-management in the medical field is used to highlight the patients' role to control their diseases [2], its application in Iraq is poor and fails to comply with the effort required to control them. However, the use of self-management and education in the healthcare field can be aided by a range of practices known as knowledge management (KM) [3].

$\mathrm{KM}$ is a dynamic process of collecting, storing, sharing and generating knowledge [4]. Essentially, a model is proposed to create knowledge, that simplifies the interaction of tacit and explicit knowledge, 
through the application of four conversion modes; socialization, externalization, combination and internalization (SECI). The SECI model is a reliable strategy in the area of KM and has been successfully implemented to manage knowledge in various domains including the healthcare sector. It can be applied to integrate $\mathrm{KM}$ and knowledge discovery $(\mathrm{KD})$ that support patients and healthcare professionals in Iraq to manage several chronic diseases such as hypertension. This integration can be accomplished through the application of many related tools [5].

$\mathrm{KD}$ uses data mining (DM) tools that contribute significantly to the healthcare sector. Additionally, it demonstrates new and valid associations/trends with the use of machine learning techniques [6]. With typical data mining applications in healthcare data involving high-risk individual' monitoring, so that the relevant data can be passed on to them [7], prediction of the length of stay of patients with acute traumatic spinal cord injury [8], and prediction of hypertension from patient's medical records along with other diseases [9]. Furthermore [10], data mining is an effective tool for handling the rapid expansion of medical knowledge and healthcare data. All the factors mentioned above are key indicators of why the Iraqi government should implement E-healthcare. In addition, the application of data mining using a SECI model will enhance medical knowledge and comprehension.

One very reliable approach in data mining is association rules mining (ARM). It is utilized to focus on interesting relationships among a set of items in a dataset. Typically to discover a group of highly cooccurring features, those are linked to a large number of transactions in a database. ARM is based on the implication of $(\mathrm{X}, \mathrm{Y})$ i.e. $\mathrm{X} \Rightarrow \mathrm{Y}$, if $\mathrm{X}$ exists then so does $\mathrm{Y}$. A derived subset of class association rules (CARs) is therefore ARM, where the consequence is limited to one target class (label) [11]. It produces classification rules based on the integration of both classification and association. The integrated paradigm of CARs proposed [12] is accomplished through the discovery of a special subset of ARs where the right-hand side of the equation is confined to the classification class label. In previous research, CARs have been used to demonstrate that classified rule patterns could be combined with a similar pattern, in the scope of; a colorectal cancer database [13], case-based reasoning. CARs have also been used in [14] to extract information concerning associations from a given case base using various approaches such as case-based reasoning $[15,16]$. Consequently, CAR as a DM tool can be used to discover significant relationships between risk factors implicated in target disease.

In this paper, the SECICAR is proposed as a new framework to overcome the barriers that have so far delayed the implementation of E-healthcare in Iraq. The SECICAR employs a SECI model that enables knowledge sharing and enhanced expertise. It can be carried out through a web portal, to bring healthcare experts, hypertensive patients in one environment. This acquired knowledge could encourage decisionmaking related to hypertension, health/lifestyle factors and other diseases. Besides, a further presented contribution is the integration of DM into a SECI Model, where initial outcomes have been promising, as the CARs have revealed that uncontrolled high blood pressure besides other symptoms can lead to complications including heart disease. To the best of our knowledge, no such integration has been conducted using CARs into the SECI model the health sector in Iraq. The score of validation shows that the SECICAR framework can disseminate appropriate knowledge. In addition, the evaluation of the web portal has revealed no major issues related to its usability.

The remaining sections of the paper is organized as follows: In Section 2, the Background includes factors that hindered the E-health in Iraq. Section 3 describes the SECI model issues. In Section 4, the details of the proposed framework is demonstrated. Section 5 presents mining the proposed model. In Sections (6, 7 ), validation and evaluation are presented and discussed. Finally, the conclusion is presented in Section 8.

\section{BACKGROUND: FACTORS HINDERING THE E-HEALTH SYSTEM IN IRAQ}

$\mathrm{KD}$ and $\mathrm{KM}$ are key components in supporting e-healthcare sectors in Arabic countries [17]. It is described as a developing field in the connection of medical informatics, public health. Whilst, the World Health Organization states that e-health is 'practice in the health area, of digital data-conveyed and processed electronically to boost healthcare services. This has been achieved in both fields at the local site and abroad. Considerable attempts have been undertaken by the Iraqi government to improve the e-health sector. Consequently, several activities have arisen leading to numerous viewpoints, extending from making electronic records for patients [18]. Nevertheless, the execution of these activities has been hindered by numerous issues as outlined in Figure 1.

a) Non-connectivity of data systems

Even though a few territorial directorates and central clinics are utilizing data frameworks [19], there has been little effort to associate or integrate these data frameworks to construct a national healthcare framework [20]. 
b) Inadequate computer skills

The computer abilities of healthcare staff and experts are inadequate for their involvement in utilizing computer applications [21]. No rules or training are given on the handling of electronic medical records (EMRs), and staffs complain about the poor upkeep of computers [22], systems and ancient terminals.

c) Non-adoption of health information services (HIS)

There are basic issues related to arranging and embracing HIS and its execution in Iraq; some are caused by poor technical support about time and budget [23].

d) Human factors

This issue is considered a major reason for the failure to adopt E-healthcare centers in Iraq. Human factors may include negative perceptions of healthcare experts towards using new technology [24]. Furthermore, patients have lost trust in staff when computer-based medical arrangements are needed. As a result, medical staff is critical of change from a traditional method to computer-based healthcare services.

e) Social and cultural factors

Cultural factors including traditions, language and religion have contributed towards the failure of ehealth implementation due to restricted human interactions [25]. Iraqi and Middle Eastern individuals are profoundly impacted by their culture and there is an inclination towards physical interaction rather than virtual contact [26].

f) Medication safety

[27] Stated that medication safety consists of two main e-health elements. First, the digital divide and communication gap among healthcare sectors, which affect medical incidents and patients' reported issues. Second, not employing the new technology effectively, results in poorly handwritten reports. This problem can be resolved using (CPOE) computerised provider order entry, but implementation is being adopted slowly.

g) Economic obstructions

The conversion of paper records to electronic datasets can be very expensive. Such a high cost may require a process of IT selection in healthcare sectors, which may result in the slow execution of e-health applications [28].

h) Security and privacy

This constraint is related to the ease of accessing patient' EMRs as some medical records cannot be revealed to others without the consent of both the specialist and patient [29].

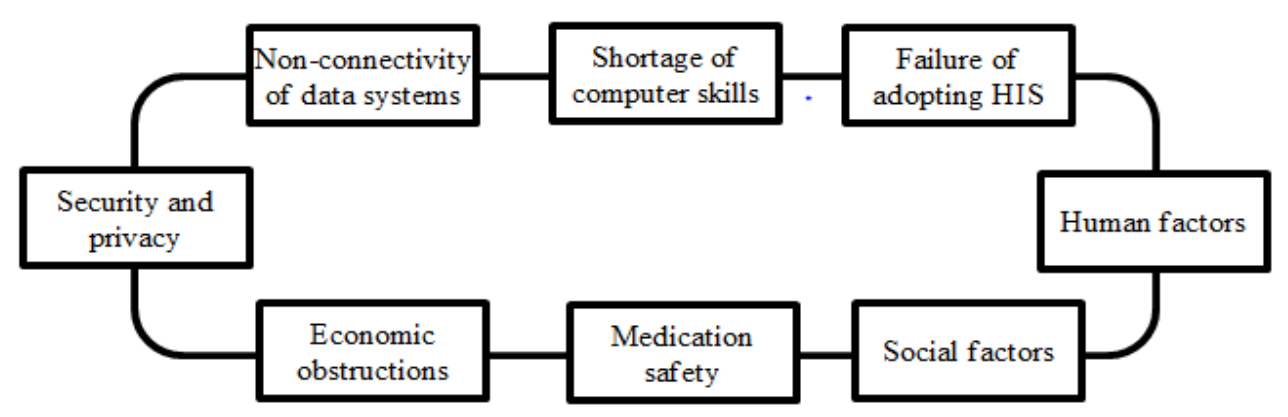

Figure 1. E-health care factors

\section{SECI MODEL ISSUES}

The SECI model is a powerful method to manage and share data. It can be implemented through the main four stages (socialization, externalization, combination, internalization) as illustrated in Figure 2 [30]. Each model may occur by using special tools, in some circumstances, it requires the knowledge conversion procedures to be carried out in the same place. Islam affects Iraqi culture by obligating moral values among people from related countries. Family is tremendously appreciated in Iraqi culture as it provides safety to its individuals. These connectives offer help, such as job opportunities, are specified to family relatives through family leaders.

The stages of externalization and internalization could be hindered in Iraqi culture [31]. Whereas, the use of the SECI sections has been examined. It has been reported that socialization and combination can be accomplished in Arab countries, such as Iraq. However, there is a tendency to withhold knowledge during communication unless colleagues are trusted. 


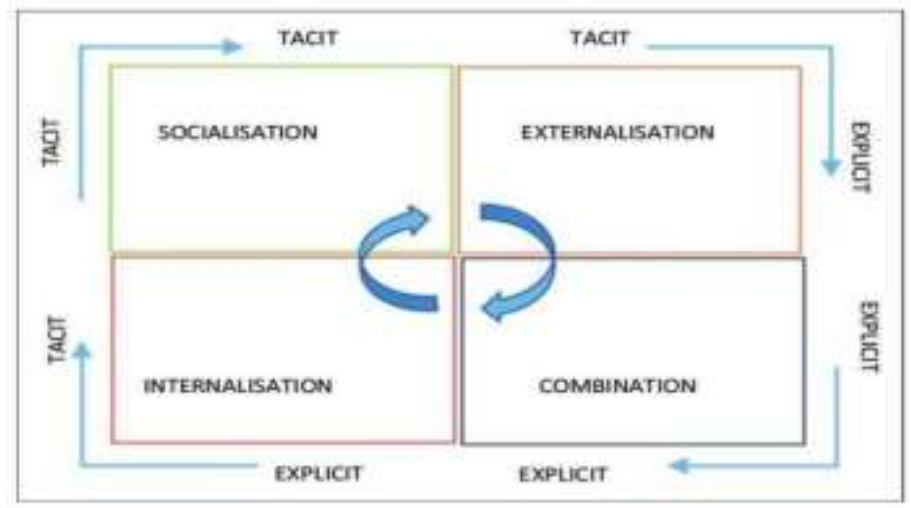

Figure 2. The SECI model [32]

This factor may influence the sharing of tacit knowledge and the externalization stage. Ultimately, the internalization stage may not function efficiently due to an absence of confidence and distrust of the data given by the knowledge holder. Therefore, the following proposals could be utilized to improve the usage of [4] the model in Iraq.

a) Socialization. Iraqi individuals are known to be pre-socialized [31]. Thus, achieving reasonable relations between employer and employee will confirm socialization in Iraqi organizations. Trust is a significant step of the SECI model, so social actions and religious activities such as festivities and graduations can strengthen trust among generations.

b) Externalisation. Office design could be improved in Iraqi organizations to enable more communication between employees. This will provide more opportunities for conversation in the work location. Encouragement plays a significant role in knowledge sharing conversations. Incentives should be focused on knowledge sharing performance among individuals. Moreover, in socialization, associations can take advantage of all occasions to upgrade trust and build connections amongst individuals thus fortifying externalization.

c) Combination. Iraqi culture in the Arabic community is known to be focused on authority and decisionmaking. A valuable key to overcome this issue would be to increase the level of contribution to decision-making. This path will enable knowledge to flow smoothly among all members regardless of their standing in the organization.

d) Internalization. Career rotation is gradually being implemented into Iraqi culture. To raise the adoption of suggested solutions, employees can be moved around, which could boost knowledge conversion and increase enthusiasm. Members of Iraqi organizations could be given opportunities to practice by attending training courses, are allocated accordingly to guarantee internalization becomes a reality.

\section{PROPOSED SECICAR}

The proposed framework highlights the Iraqi context, which is strongly impacted by Arabic culture when implementing the SECI model [31]. It is mainly designed to deal with the barriers described and the prospective of technological mining tools. It might assist doctors to find proper solutions in the combination step of the SECI model as shown in Figure 3. The proposed framework, known as an E-health knowledge management system, is originally planned to address healthcare issues in Iraq, specifically the hypertension domain. As mentioned previously, the barriers outlined in Figure 2 can be categorized into four parts.

First, the Business factor constraints on handling barriers related to the failure of adopting health information services and medical safety, for instance low technical support to access medical records. Secondly, Human factors that deal with barriers connected to the usage of technology by healthcare employees, as well as cultural barriers from a patient's point of view. This will identify the negative beliefs of doctors and patients connected to the use of electronic contact with technological services, which could be a serious barrier to the success of the proposed framework.

Thirdly, the technology factor that controls the non-connectivity matters and focuses on technical skills, security and privacy. These factors involve training scopes to determine the limitation/shortage of computer skills among healthcare workers, doctors and their patients. Lastly, financial factors that attempt to focus on the restrictions and policies related to the application of healthcare information services. In other words, the high cost of converting patients' paper records to a database. 
Therefore, the suggested framework will examine these difficulties, to offer solutions to the community through a devoted knowledge management system. For this purpose, knowledge will be continually converted and created as (C, JD, P, Data miner) practice, collaboration, interaction and learning, whilst considering the technological barriers and proposing solutions to overcome this problem. As shown in Figure 3, the framework keeps the information tacit to tacit, when knowledge is passed on through $\mathrm{P}$ to the $\mathrm{Dr}$ in the first step of socialization. JD will discuss with $\mathrm{P}$ tries to find the best diagnosis but the information transfer will not be converted. Second, the externalization step starts when tacit knowledge is codified into documents for the database. C, JD, P and data miners will all have initial discussions, share information in one electronic environment so that it can be spread easily. The information and diagnosis can be linked to all parties in one portal, with info transfer from tacit to explicit. Third, the simplest form of the combination step is explicit to tacit; the patient records will be systemized through technology. The sources of knowledge are codified to create new knowledge. Then, C \& JD will have a final discussion; the data miner processes the information to be ready for the DM box. After C \& JD have discussed the matter, the Data miner can then extract the best CARs to control risk factors for optimal advice.

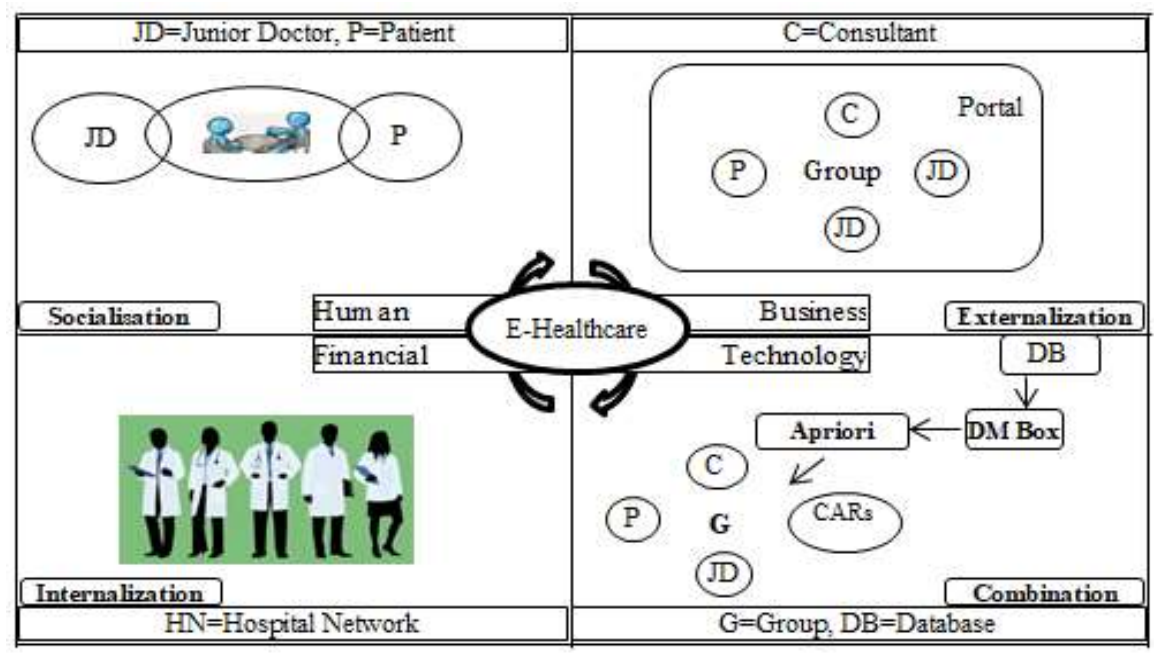

Figure 3. The proposed SECICAR

Finally, as explicit sources are used and learned, the knowledge is internalized, modifying the parties existing tacit knowledge. In other words, all JDs involved learn from C, then the knowledge goes into a spiral model to teach other JDs, with DM tools used to support decision making. Ultimately, the proposed framework will resolve some of the problems mentioned and propose solutions, which will be made available to the community via a knowledge management system.

This strategy will be utilized to support the hypertension community, which is growing at an alarming rate in Iraq. By considering the problems faced through testing protocols, interviews, data mining analyses extracted from the dataset and the use of mining algorithms association rules, the typical issues and problems faced can be elicited from the hypertension community linked to the four parts presented in Figure 3. Consequently, the newly learned knowledge can then be recommended to support both hypertensives and healthcare professionals. The aim of the proposed strategy is not only to provide related knowledge to healthcare professionals pursuing best practices, strategies, and information sharing but also to patients seeking contact with professionals or healthcare services. The suggested approach will also allow access to recommended academic papers associated with specific problems, thus keeping healthcare professionals up to date with new results.

\subsection{Design of web portal}

The design of the portal involves the representation of the website components in a graphical way to create the website. It includes a forum that provides socialization between different participants in an online environment. It is also linked with one of the most visited hypertension web forums in the world: WHOHypertension. In this forum, different individuals suffering from hypertension can meet and learn from each other about guidelines and best practices. The interface of the Web Portal as shown in Figure 4. The portal contains: 
a) About: A short description and goals of the web portal. Description of Iraq's high blood pressure and high blood pressure. How can risk factors lead to high blood pressure and how class rules can help resolve this problem.

b) A forum: The forum enables socialization to hypertensives to communicate with others. It also offers support related to hypertension and disseminates experiences concerning the disease. In addition, there is a link to discuss different related health issues. It is possible to share experiences, thoughts, tips and guidelines on hypertension management.

c) Official documents: The official document section offers guidelines, strategies associated to hypertension, and self-management from trusted sources for hypertension visitors of SECICAR. This page applies the combination mode as it integrates different explicit knowledge from different sources.

d) Hypertension data: The hypertension dataset provides externalization to open sources for supporting future studies concerning hypertension problem-solving. They are extracted from an optional survey offered on the same website. Therefore, this applies to the externalization mode as it externalizes hidden information to other visitors.

e) Hypertension videos: Hypertension videos consist of several self-management, educational best practices and guidelines. They are provided to determine various best practices in health complications, including symptoms of hypertension, exercise, medication and appropriate diet. The hypertension videos offer great internalization opportunities, as visitors can learn from the provided videos and practicition.

f) FAQs: Frequently asked questions about hypertension and risk factors. This page facilitates the externalization of self-management of hypertensives among all consultants, junior doctors and hypertensives.

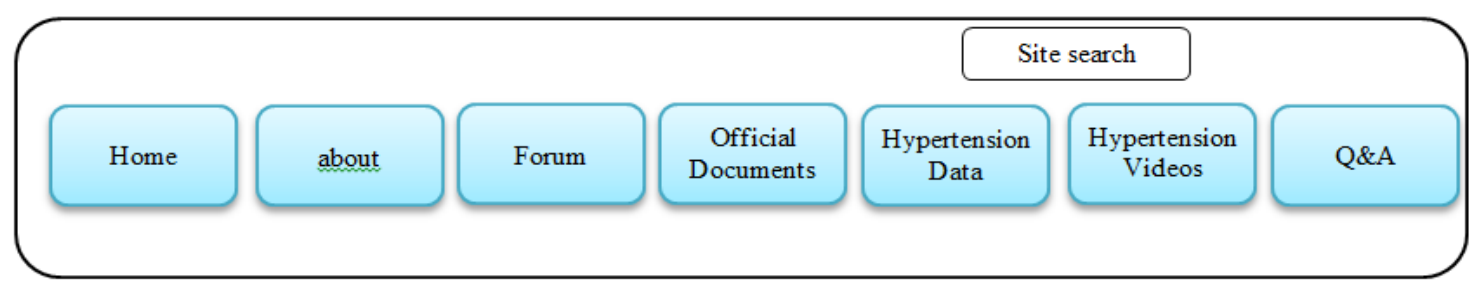

Figure 4. The interface of the web portal

\section{MINING SECI MODEL SECICAR}

The mining SECI model (SECICAR) is clarified through a simple medical diagnosis scenario, where a hypertensive might be exposed to another disease based on risk factors. A hypertensive would attend hospital suffering from some problems (diabetes, cholesterol, or thalassemia). The mining stage would be to find CARs between these symptoms and another disease. Therefore, the heart disease dataset has been chosen from a UCI repository contains the required risk factors, consisting of 334 records. 73 participants are asked to provide demographic information about themselves such as (gender, age, education level, computer skills to check whether they can participate or not, as well as the below attributes). The attributes' values of the dataset used are as follows: $\{$ age $=(29-77)$, sex $=$ (male or female), chest pain type values $(1,2,3,4)$, blood pressure (normal, prehypertension, stage1 hypertension, stage 2 hypertension). Serum cholesterol mg/dl (< $200=$ normal, $200-400=$ high, $>400=$ very high $)$, fasting blood sugar $(<120=$ true, $>120=$ false $)$, resting electrocardiographic result values $(0,1,2)$, maximum heart rate achieved (71-200), exercise induced angina (yes, no), oldpeak=ST depression induced by exercise relative to rest, the slope of the peak exercise ST segment $(0,1,2)$, number of major vessels $(0,1,2,3)$ coloured by fluoroscopy, thalassemia $(0=$ normal; $1=$ fixed defect; $2=$ reversible defect).

The Dependency Analyses, specifically association rules, the Apriori algorithm were chosen to explore association rules between hypertensive and other diseases such as heart disease via a laboratory medical test. CAR is an approach of Apriori, which has been adopted in this study to find CARs in large amounts of data.

In the initial mining attempt, the minimum support (MS) is set at 0.1 , the minimum confidence (MC) at $0.9(90 \%)$ to ensure that only rules with high accuracy are produced. However, few rules are produced. As a result, the minimum support value is modified experimentally until the required rules are generated from a dataset at $\mathrm{MS}=0.1, \mathrm{MC}=0.4$. In Table 1 , the Rules are organized by the level of confidence, i.e. the rule which was initially extracted is listed first, as follows: 
Table 1. Best class association rules

\begin{tabular}{|c|c|c|c|}
\hline $\begin{array}{c}\text { Rule } \\
\text { No }\end{array}$ & Best Rules & $\begin{array}{c}\text { Minimum } \\
\text { Support }\end{array}$ & Confidence \\
\hline 1 & sex=female ChestPain=two 35=>HeartDisease=yes 34 conf:(0.97) & 0.1 & 0.9 \\
\hline 2 & sex $=$ male ChestPain=zero $c a($ flourosopy $)=$ one $32=>$ HeartDisease $=$ no 31 conf: $(0.97)$ & 0.1 & 0.9 \\
\hline 3 & testBPS $=$ PreHypn chol=high $\frac{\text { fbs }>120=\text { FALSE } \text { ca(flourosopy })=\text { zero } 35=>\text { HeartDisease }=y e s}{32 \text { conf: }(0.91)}$ & 0.1 & 0.9 \\
\hline 4 & $\begin{array}{c}\text { testBPS=PreHypn chol=high exang=no ca(flourosopy })=\text { zero } 33=>\text { HeartDisease }=\text { yes } 31 \\
\text { conf: }(0.94)\end{array}$ & 0.1 & 0.9 \\
\hline 5 & $\begin{aligned} \text { testBPS }=\text { PreHypn chol=high fbs }<120=\text { FALSE oldpeak }='(- \text { inf }-0.62]^{\prime} 38=>\text { HeartDisease }=y e s \\
32 \text { conf: }(0.84)\end{aligned}$ & 0.1 & 0.9 \\
\hline 6 & age $=$ '(57.8-62.6]' ChestPain=zero 37 $\Rightarrow$ HeartDisease $=$ no 31 conf: $(0.84)$ & 0.1 & 0.8 \\
\hline 7 & $\mathrm{ca}($ flourosopy $)=$ two $38=>$ HeartDisease $=$ no 31 conf: $(0.82)$ & 0.1 & 0.8 \\
\hline 8 & $\begin{aligned} \text { testBPS }=\text { PreHypn fbs }<120 & =\text { FALSE exang }=\text { no ca(flourosopy })=\text { zero thal }=\text { FixedDefect } \\
34 & =>\text { HeartDisease }=\text { yes } 33 \text { conf: }(0.97)\end{aligned}$ & 0.1 & 0.8 \\
\hline 9 & $\begin{array}{c}\text { testBPS }=\text { PreHypn fbs }<120=\text { FALSE ca(flourosopy)=zero thal=FixedDefect } \\
38=>\text { HeartDisease }=\text { yes } 36 \text { conf: }(0.95)\end{array}$ & 0.1 & 0.8 \\
\hline 10 & $\begin{aligned} \text { testBPS }=\text { PreHypn fbs }<120 & =\text { FALSE exang }=\text { no oldpeak }=\text { '(-inf }-0.62]^{\prime} \text { thal=FixedDefect } \\
34 & =>\text { HeartDisease }=\text { yes } 32 \text { conf: }(0.94)\end{aligned}$ & 0.1 & 0.8 \\
\hline 11 & $\begin{array}{c}\text { testBPS=PreHypn fbs }<120=\text { FALSE exang=no ca(flourosopy) }=\text { zero } 40=>\text { HeartDisease }=\text { yes } \\
37 \text { conf: }(0.93)\end{array}$ & 0.1 & 0.8 \\
\hline 12 & $\begin{aligned} \text { testBPS }=\text { PreHypn fbs }<120 & \left.=\text { FALSE exang=no oldpeak='(-inf }-0.62]^{\prime} \text { ca(flourosopy }\right)=\text { zero } \\
34 & =>\text { HeartDisease }=\text { yes } 31 \text { conf: }(0.91)\end{aligned}$ & 0.1 & 0.8 \\
\hline 13 & $\begin{array}{c}\text { testBPS }=\text { PreHypn fbs }<120=\text { FALSE oldpeak }='(- \text { inf }-0.62] \text { ' thal=FixedDefect } \\
39=>\text { HeartDisease=yes } 35 \text { conf: }(0.9)\end{array}$ & 0.1 & 0.8 \\
\hline 14 & 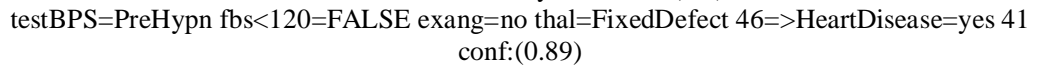 & 0.1 & 0.8 \\
\hline 15 & $\begin{array}{c}\text { testBPS=PreHypn fbs }<120=\text { FALSE oldpeak }='(- \text { inf }-0.62]^{\prime} \text { ca(flourosopy)=zero } \\
40=>\text { HeartDisease=yes } 35 \text { conf: }(0.88)\end{array}$ & 0.1 & 0.8 \\
\hline 16 & $\begin{array}{c}\left.\text { testBPS }=\text { PreHypn fbs }<120=\text { FALSE oldpeak }=\text { '(-inf- } 0.62]^{\prime} \text { ca(flourosopy }\right)=\text { zero } \\
40=>\text { HeartDisease=yes } 35 \text { conf: }(0.88)\end{array}$ & 0.1 & 0.8 \\
\hline 17 & $\begin{array}{l}\text { testBPS }=\text { PreHypn fbs }<120=\text { FALSE exang=no oldpeak='(-inf- } 0.62]^{\prime} 43=>\text { HeartDisease }=y e s \\
37 \text { conf: }(0.86)\end{array}$ & 0.1 & 0.8 \\
\hline 18 & $\begin{array}{c}\text { testBPS }=\text { PreHypn fbs }<120=\text { FALSE thal }=\text { FixedDefect } 54=>\text { HeartDisease }=\text { yes } 45 \\
\text { conf: }(0.83)\end{array}$ & 0.1 & 0.8 \\
\hline 19 & $\begin{array}{c}\text { testBPS }=\text { PreHypn fbs }<120=\text { FALSE ca(flourosopy) }=\text { zero } 49=>\text { HeartDisease }=\text { yes } 41 \\
\text { conf: }(0.84)\end{array}$ & 0.1 & 0.8 \\
\hline 20 & testBPS $=$ PreHypn chol=high ca(flourosopy $)=$ zero $40=>$ HeartDisease $=$ yes 35 conf: $(0.88)$ & 0.1 & 0.8 \\
\hline 21 & $\begin{array}{c}\text { testBPS=PreHypn fbs }<120=\text { FALSE oldpeak='(-inf-0.62]' 52=>HeartDisease=yes } 42 \\
\text { conf: }(0.81)\end{array}$ & 0.1 & 0.8 \\
\hline 22 & $\begin{aligned} \text { testBPS }=\text { PreHypn chol=high exang } & =\text { no oldpeak }={ }^{\prime}(- \text { inf }-0.62]^{\prime} 36=>\text { HeartDisease }=y e s \\
& \text { 31 conf: }(0.86)\end{aligned}$ & 0.1 & 0.8 \\
\hline 23 & $\begin{array}{c}\text { testBPS=PreHypn chol=high exang= }=\text { no thal=FixedDefect } 36=>\text { HeartDisease }=\text { yes } 31 \\
\text { conf: }(0.86)\end{array}$ & 0.1 & 0.8 \\
\hline 24 & testBPS $=$ PreHypn chol=high $\frac{\text { fbs }<120=\text { FALSE oldpeak }='(- \text { inf }-0.62]^{\prime} 38=>\text { HeartDisease }=y e s}{32 \text { conf: }(0.84)}$ & 0.1 & 0.8 \\
\hline 25 & testBPS=PreHypn chol=high thal=FixedDefect $43=>$ HeartDisease=yes 36 conf:(0.84) & 0.1 & 0.8 \\
\hline 26 & testBPS=PreHypn chol=high oldpeak='(-inf-0.62]' 47=>HeartDisease=yes 37 conf: $(0.79)$ & 0.1 & 0.4 \\
\hline 27 & testBPS=PreHypn chol=high fbs $<120=$ FALSE exang=no $43=>$ HeartDisease $=$ yes 32 & 0.1 & 0.4 \\
\hline 28 & testBPS=PreHypn chol=high exang=no 52=>HeartDisease=yes 37 conf: $(0.71)$ & 0.1 & 0.4 \\
\hline 29 & 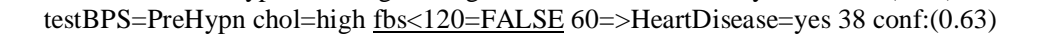 & 0.1 & 0.4 \\
\hline 30 & testBPS=PreHypn $109=>$ HeartDisease=yes 61 conf:(0.56) & 0.1 & 0.4 \\
\hline 31 & testBPS=PreHypn $109=>$ HeartDisease $=$ no 48 conf:(0.44) & 0.1 & 0.4 \\
\hline
\end{tabular}

In group 1, some Rules are filtered out from the list of CARs for instance $(1,2,6,7)$. Rule 1 reveals that two factors (sex=female, ChestPain=two) could be associated with heart disease. However, the factors could also be associated with another disease such as injured ribs or asthma. Rules $(2,6 \& 7)$ show all risk factors do not include a testBPS and label=no, so they are not in the scope of the hypertensives.

Group 2, rules $(30,31)$ contained testBPS=PreHypn, implicated HeartDisease (yes, no) with low conf:(0.44, 0.56). In addition, they associated to two different labels of Heart Disease (yes, no). Therefore, such rules cannot be considered due to different classes.

Group 3, the rules related to factors (testBPS=PreHypn, chol=high) are extracted in $(4,20,22,26$, 28) associated to HeartDisease=yes. The highest confidence is attributed to 0.94 in rule 4 and followed by the confidence $(0.88,0.86,0.79,0.71)$ of the associated rules to this correlation, which show a strong relationship between high blood pressure and high cholesterol.

Group 4, the next strongest rules contain three factors (testBPS=PreHypn, chol=high, fbs $>120=F A L S E)$, are extracted in $(3,5,24,27,29)$ and display their association to HeartDisease=yes. The 
highest confidence is attributed to 0.91 in rule 3 and followed by confidence $(0.84,0.84,0.74,0.63)$. This reveals that there is a strong relationship between hypertension, high cholesterol and diabetes.

Group 5, the rules contain three factors (testBPS=PreHypn, chol=high, thal=FixedDefect), which are mined frequently in $(23,25)$ associated to HeartDisease=yes, the confidence is $(0.86,0.84)$. This confirms the relation between hypertension \& cholesterol as derived in Groups $3 \& 4$, in addition to thalassemia.

Group 6, the next strongest rules relate to (testBPS=PreHypn, fbs $>120=$ FALSE), are extracted in $(11,12,15,16,17,19,21)$ display their association to class HeartDisease=yes. The highest confidence is attributed to 0.93 in Rule 3 , followed by $(0.91,0.88,0.88,0.86,0.84,0.81)$ of the associated rules to this correlation. This reveals that there is a strong association between hypertension and diabetes.

The final strongest rules are in Group 7 which relate to (testBPS=PreHypn, fbs $>120=$ FALSE, thal=FixedDefect) are extracted in $(8,9,10,13,14,18)$ indicating their association to HeartDisease=yes. The confidence is $(0.97,0.95,0.94,0.90,0.89,0.83)$ which confirms the rules of Groups $3,4 \& 5$, which have a strong relation between hypertension \& diabetes with thalassemia.

The results of mining SECICAR reveal interesting CARs among groups associated with heart disease. The rules contain two factors (testBPS=PreHypn, chol=high), which appeared twelve times in groups (3, 4 \& 5) frequently revealing a strong association between hypertension and cholesterol. In addition, the rules contain a combination of (testBPS=PreHypn, fbs $>120=$ FALSE), which appeared eighteen times in groups $(4,6,7)$ showing a strong correlation between hypertension and diabetes, which could lead to heart disease. Moreover, interesting CARs may be found in groups $(5,7)$ where factors appeared eight times in CARs i.e. (testBPS=PreHypn, thal=FixedDefect) showing other symptoms may implicate heart disease. As regards to rules that contain three factors respectively (testBPS=PreHypn, chol=high,fbs $>120=$ FALSE), (testBPS=PreHypn, chol=high, thal=FixedDefect), (testBPS=PreHypn, fbs $>120=$ FALSE and thal=FixedDefect)], they appeared (five, two and six) times in groups (4,5 and 7) which demonstrates association among the mentioned factors, that could lead to heart disease. The newly discovered CARs are to be matched with medical advice to make hypertensives aware of the risk that uncontrolled factors adherent to hypertension could lead also to heart disease.

\section{SECICAR VALIDATION}

The process of validation was to determine whether the model accurately described the real world from the viewpoint of the planned uses of the model [33]. The validation of the proposed framework was conducted with nine intended participants of three different types: (1 Consultant, 2 JDs), (1 Data miner, 1 IT professional) and (4 hypertensives). The workshop lasted about four hours in total for the validation and evaluation parts in a primary healthcare centre (PHC) in Iraq as follows:

a) The aim, objectives and explanation of the framework are given through a presentation. In addition, a description of the framework and its components was demonstrated to all participants.

b) After the presentation, the knowledge and results associated with the technological barriers of Iraqi hypertension citizens were discussed.

c) Participants were asked to pose questions regarding the framework and comment on the validation form.

The form contained statements relating to ethical approval. The consultant and JDs were also questioned to determine their years of experience in healthcare. All participants should answer multiplechoice questions. They needed to specify their choices with values ranging: strongly agree $(\mathrm{SA})=1$, agree $(A)=2$, neutral $(N)=3$, disagree $(D)=4$, strongly disagree $(S D)=5$. The range $5-1=4$, length of range $4 / 5=0.8$, the weight of responses were $(\mathrm{SA}=1-1.80, \mathrm{~A}=1.81-2.60, \mathrm{~N}=2.61-3.40, \mathrm{D}=3.41-4.20, \mathrm{SD}=4.21-5)$ as illustrated in Table 3. Table 2 summarises a profile of the participants, who demonstrated adequate expertise in the field of computing and acceptable knowledge in the area of DM. A set of statements were given and the participants were asked whether they agreed or disagreed with each one.

Table 2. The profile of participants

\begin{tabular}{ccccccc}
\hline Participant Group & Participant & \multicolumn{5}{c}{ Years of Experience } \\
& ID & $1-5$ & $6-10$ & $11-15$ & $16-20$ & $>20$ \\
\hline Hypertensives & P1 & $\sqrt{ }$ & & & & \\
& P2 & $\sqrt{ }$ & $\sqrt{ }$ & & \\
& P3 & & $\sqrt{ }$ & & $\sqrt{ }$ & \\
Consultant & P4 & & & & & \\
Junior Doctor & C1 & $\sqrt{ } 1$ & $\sqrt{ }$ & & & \\
& JD2 & $\sqrt{ }$ & & $\sqrt{ }$ & \\
Data Miner & DMr & & & & & $\sqrt{ }$ \\
IT Professional & IT & & & &
\end{tabular}


The first statement ( $\mathrm{S} 1$ ) focused on hypertension disease in Iraq, 8 participants agreed, 1 JD strongly agreed. The mean of $(\mathrm{S} 1)=($ Total score/number of participants $=(1 * 1+8 * 2+0 * 3+0 * 4+0 * 5) / 9)$, the mean $=1.88$ in the range of (Agree) as shown in Table 3. (S2) to what extent the integrated framework deals with appropriate issues related to hypertensives, showed that 8 participants agreed, 1 patient was neutral, the mean=2.11. (S3) tested knowledge of mining capabilities related to our integrated framework to assess whether the framework had captured new knowledge related to hypertension. 1 consultant, 2 JDs agreed. The rest are strongly agreed, the mean=1.33. (S4) examined whether the guidelines, best practices, policies, strategies and governance could assist hypertensives in Iraq with technology-related complications. 1 JD agreed, 1 patient was neutral. The rest are strongly agreed with this statement, the mean=2. (S5) focused on knowledge dissemination dimension measures and whether the framework provided a mechanism to share useful knowledge with hypertensives and healthcare professionals. 1 JD was neutral, 1 patient disagreed, others agreed, the mean=2.33. (S6) measured whether our integrated framework facilitates the sharing of tacit strategies among different individuals. $1 \mathrm{JD}, 1$ patient,1 IT agreed, others strongly agreed, the mean=1.55. (S7) focused on whether the framework facilitated the sharing of different explicit policies among different individuals. $1 \mathrm{JD}, 1$ patient, 1 IT strongly agreed, others agreed, the mean=1.70. (S8) focused on knowledge dissemination field measures, and to what extent the framework supports the conversion of explicit best practices into tacit knowledge. 1 patient, 1 IT agreed. The consultant, 1 JD were neutral, others strongly agreed, the mean $=1.66$.

Finally, the validation form contained one open-ended statement where participants could comment on and suggest any further issues. The consultant suggested that a healthcare specialist should get involved with JDs in the same electronic environment to expand and encourage knowledge sharing. The average for all means is (1.82), which is close to the range of Agree. The scores indicate that most participants were satisfied with the framework support of E-health, the knowledge extraction and the ability of the framework to disseminate appropriate knowledge.

Table 3. The frequency of participants

\begin{tabular}{cccccc}
\hline Item & $\begin{array}{c}\mathrm{SA}=1 \\
\mathrm{R}=1-1.80\end{array}$ & $\begin{array}{c}\mathrm{A}=2 \\
\mathrm{R}=1.81-2.60\end{array}$ & $\begin{array}{c}\mathrm{N}=3 \\
\mathrm{R}=2.61-3.40\end{array}$ & $\begin{array}{c}\mathrm{D}=4 \\
\mathrm{R}=3.41-4.20\end{array}$ & $\begin{array}{c}\mathrm{SD}=5 \\
\mathrm{R}=4.21-5\end{array}$ \\
\hline S1 & 1 & 8 & 0 & 0 & 0 \\
S2 & 0 & 8 & 1 & 0 & 0 \\
S3 & 6 & 3 & 0 & 0 & 0 \\
S4 & 1 & 7 & 1 & 0 & 0 \\
S5 & 0 & 7 & 1 & 1 & 0 \\
S6 & 5 & 3 & 1 & 0 & 0 \\
S7 & 3 & 6 & 0 & 0 & 0 \\
S8 & 5 & 2 & 2 & 0 & 0 \\
\hline
\end{tabular}

\section{EVALUATION OF THE WEB PORTAL}

The evaluation was concerned with testing the designed web portal and whether it has met the set objectives of this research [34]. The heuristic methodology is an effective strategy used to examine usability issues [35], which can be evaluated from ten scopes of heuristics statements; as illustrated in Table 4. The evaluation procedure is started by assigning the same participants from the validation workshop on SECICAR as shown in Table 2. The participants were asked to familiarise themselves with the portal initially. They were also asked to grant their ethical approval to participate in this evaluation study. A summary of the participants' feedback shown in Table 4.

The first heuristic shows that $77.78 \%$ indicated that there were no usability issues with regards to visibility of the system status, whereas two patients indicated a cosmetic issue. The second heuristic revealed that more than half the participants $(55.50 \%)$ agreed that there was no issue to the factor of a match between the system and the real world. While three participants reported a cosmetic problem and one participant indicated a minor problem. The third and fourth heuristics showed that both factors (user control and freedom) and (consistency and standards), resulted in $77.78 \%$ choosing no issues with any usability of the web portal. However, two participants, one IT and one Data miner indicated cosmetic issues. The fifth heuristic resulted in just over half $(55.50 \%)$ agreeing that there were no issues with error prevention. In addition, two JDs, one IT specialist, one patient indicated a cosmetic usability issue. The sixth heuristic revealed that around two-thirds of the respondents agreed that there were no issues with recall in the web portal. However, one IT indicated a minor problem two patients pointed out a cosmetic problem. For the seventh heuristic, $77.78 \%$ indicated that there was no usability issue with regards to flexibility and efficiency, whereas one JD indicated a cosmetic problem, one consultant indicated a minor problem. The eighth heuristic resulted in half $(55.50 \%)$ of the respondents agreeing that there were no issues with regard to error 
indication and recovery. While, two JDs indicated cosmetic issues, one IT, one data miner pointed out a minor problem. Finally, for the ninth heuristic, $88.89 \%$ indicated that there was no issue with help and documentation, while one respondent reported a cosmetic problem. In summary, according to the evaluation results, there are no major issues related to the usability of the web portal.

Table 4. Evaluation of the web portal

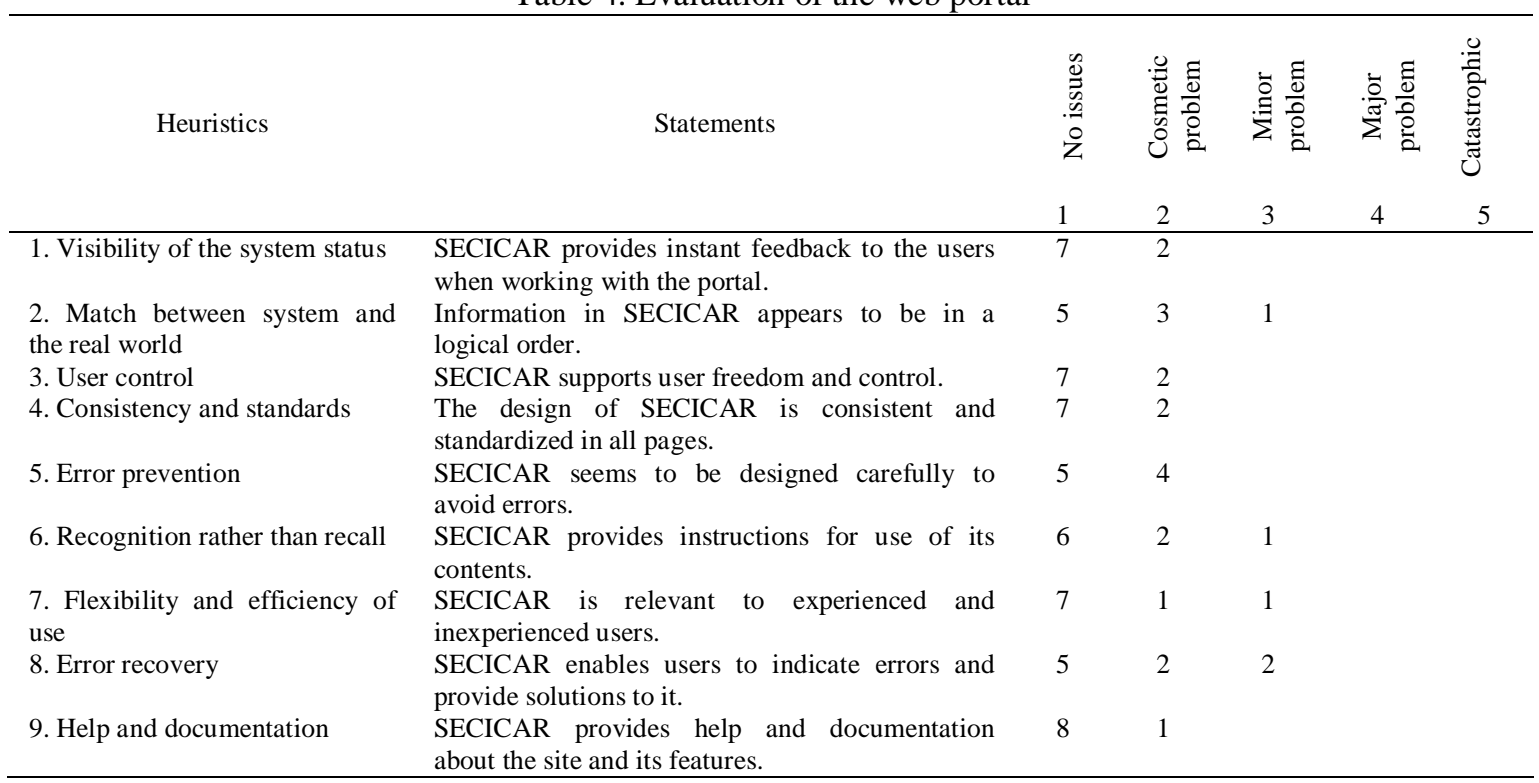

\section{CONCLUSION}

This paper has pinpointed the main barriers that have delayed the implementation of E-health in Iraq. The SECICAR framework is proposed to overcome technological barriers by integrating CARs into the SECI model and applied to hypertensives. The new framework suggested a web portal that assembles Consultants, JDs, Patients and CARs in an electronic environment to share knowledge and experience. The portal reflects the SECI model steps because pages represent socialization, externalization, combination and internalization for extracting useful CARs. The extracted CARs in Groups $(3,4,5,6 \& 7)$ form a reliable dataset, which displays a strong association among (hypertension, diabetes, cholesterol \& thalassemia) as risk factors that could lead to heart disease. The mined CARs are to be matched with medical advice to control risk factors. The integrated framework was validated through a workshop in a PHC in Iraq. Most participants expressed their satisfaction with the framework and its components. Only one participant suggested the involvement of a specialist in the same environment to share knowledge. Following this, an evaluation of the web portal was conducted by the same participants. The results show that there are no major issues related to the usability of the web portal and each mode of the SECI model. The average of all means of validation is equal to 1.82, which indicates the level of agreement (Agree). Some limitations have been recognized in the research i.e. lack of DM experts in Iraq. This might have an impact on the analysis of our proposed framework and the web portal evaluation. Technically, this has led us to include less number of data miners and participants with sufficient computer expertise. One other limitation of the current research is the fact that the respondents involved in this research come from Iraq, while the main dataset is taken from the UCI repository. Ultimately, due to these promising findings, further work on employing more mining algorithms will continue with the aim of presenting a more optimal framework.

\section{ACKNOWLEDGEMENTS}

A special thank to all who participated in this research, we also want to deliver our gratitude to PHC to provide a great environment to fulfill the aim of this study.

\section{REFERENCES}

[1] B. W. H. Bdair et al., "Cardiovascular Risk Factors for Hypertension and Diabetes among Overweight and Obese Adolescents in the City of Kerbala, Iraq," J. Cardiovasc. Dis. Res., vol. 11, no. 2, pp. 32-39, 2020. doi: $10.31838 /$ jcdr.2020.11.02.07.

Pushing towards ehealth for iraqi hypertensives: an integrated class association rules... (Ahmed Aljuboori) 
[2] J. Jiang and A.-F. Cameron, "IT-Enabled Self-Monitoring for Chronic Disease Self-Management: An Interdisciplinary Review.," MIS Q., vol. 44, no. 1, 2020. doi: 10.25300/MISQ/2020/15108.

[3] A. van der Hout et al., "Role of eHealth application Oncokompas in supporting self-management of symptoms and health-related quality of life in cancer survivors: a randomised, controlled trial," Lancet Oncol., vol. 21, no. 1, pp. 80-94, 2020. doi: 10.1016/S1470-2045(19)30675-8.

[4] I. Nonaka and H. Takeuchi, "The knowledge-creating company: How Japanese companies create the dynamics of innovation," Long Range Plann., vol. 4, no. 29, p. 592, 1996.

[5] S. Almuayqil, A. S. Atkins, and B. Sharp, "Integrated Framework of Knowledge Management and Knowledge Discovery to Support E-Health for Saudi Diabetic Patients," Int. J. Adv. Life Sci., vol. 7, no. 3, pp. 111-121, 2015.

[6] U. Fayyad, G. Piatetsky-Shapiro, and P. Smyth, "From data mining to knowledge discovery in databases," AI Mag., vol. 17, no. 3, p. 37, 1996. doi: 10.1609/aimag.v17i3.1230.

[7] M. L. Z. Alkaragole and S. Kurnaz, "Comparison of Data Mining Techniques for Predicting Diabetes or Prediabetes by Risk Factors,” 2019. doi: 10.1016/j.kjms.2012.08.016.

[8] G. Gour-Provencal, J.-M. Mac-Thiong, D. E. Feldman, J. Bégin, and A. Richard-Denis, "Decreasing pressure injuries and acute care length of stay in patients with acute traumatic spinal cord injury," J. Spinal Cord Med., pp. 1-9, 2020. doi: 10.1080/10790268.2020.1718265.

[9] C.-D. Chang, C.-C. Wang, and B. C. Jiang, "Using data mining techniques for multi-diseases prediction modeling of hypertension and hyperlipidemia by common risk factors," Expert Syst. Appl., vol. 38, no. 5, pp. 5507-5513, 2011. doi: 10.1016/j.eswa.2010.10.086.

[10] G. M. Kisumano and J. I. Masinda, "Predictors of Knowledge Management in Healthcare Organizations in Butembo," Int. Forum J., vol. 23, no. 1, pp. 53-75, 2020.

[11] M. Azmi, G. C. Runger, and A. Berrado, "Interpretable regularized class association rules algorithm for classification in a categorical data space," Inf. Sci. (Ny)., vol. 483, pp. 313-331, 2019. doi: 10.1016/j.ins.2019.01.047.

[12] B. Ma, W. Liu, and Y. Hsu, "Integrating classification and association rule mining," in Proceedings of the 4th Knowledge Discovery and Data Mining, 1998.

[13] J. A. Delgado-Osuna, C. García-Martínez, J. Gómez-Barbadillo, and S. Ventura, "Heuristics for interesting class association rule mining a colorectal cancer database," Inf. Process. Manag., vol. 57, no. 3, p. 102207, 2020. doi: 10.1016/j.ipm.2020.102207.

[14] A. S. Aljuboori, F. Meziane, and D. J. Parsons, "A new strategy for case-based reasoning retrieval using classification based on association," in 12th MLDM International Conference, 2016, p. pp 326-340. doi: 10.1007/978-3-319-41920-6_24.

[15] A. Aljuboori, "Enhancing case-based reasoning retrieval using classification based on associations," 2016 6th Int. Conf. Inf. Commun. Manag., pp. 52-56, 2016. doi: 10.1109/INFOCOMAN.2016.7784214.

[16] A. S. Aljuboori, F. Coenen, M. Nsaif, and D. J. Parsons, "Performance of case-based reasoning retrieval using classification based on associations versus Jcolibri and FreeCBR: a further validation study," in Journal of Physics: Conference Series, 2018, vol. 1003, no. 1, p. 12130. doi: 10.1088/1742-6596/1003/1/012130.

[17] S. Almuayqil, A. ATKINS, and B. Sharp, "Knowledge management framework for e-healthcare in Saudi Arabia," 2015.

[18] D. Landsbergen Jr and G. Wolken Jr, "Realizing the promise: Government information systems and the fourth generation of information technology," Public Adm. Rev., vol. 61, no. 2, pp. 206-220, 2001. doi: 10.1111/00333352.00023.

[19] T. J. Abaas, A. S. Shibghatullah, and M. M. Jaber, "Use Information Sharing Environment Concept to Design Electronic Intelligence Framework for Support E-Government: Iraq as Case Study,” Adv. Comput., vol. 4, no. 1, pp. 22-24, 2014.

[20] R. F. Albadri, M. D. Alsallal, A. S. Abdulsatar, and Q. H. Abbas, "The Readiness of E-Government Adoption in Iraq: A Case Study of Al-Muthanna Province," Int. J. Psychosoc. Rehabil., vol. 24, no. 04, 2020. doi: 10.37200/IJPR/V24I4/PR2020380.

[21] H. Al-Hashimy, A. Hamoud, and F. Hussain, "The Effect of Not Using Internet of Things in Critical life Situations in the Health Field and the Effect on Iraqi Profitability: Empirical Study in Basra," J. Southwest Jiaotong Univ., vol. 54, no. 4, 2019. doi: 10.35741/issn.0258-2724.54.4.2.

[22] Q. N. AL-Mahwey and H. R. Sayah, "The Impact of Information and Communication Technology on the Technical Quality of Health Services" A Study at Al-Shatrah General Hospital-Dhi-Qar, Iraq"," Glob. J. Manag. Bus. Res., 2020.

[23] A. J. Al-Mosawi, "Iraq Healthcare system: An update," Lupine Online J. Med. Sci. (ISSN 2641-1725), vol. 4, no. 3 , pp. 404-411, 2020. doi: 10.32474/LOJMS.2020.04.000190.

[24] H. H. ABDULQADER and A. T. SAADI, "The distribution of pathogens, risk factors and their antimicrobial susceptibility patterns among post-surgical site infection in Rizgari Teaching Hospital in Erbil/Kurdistan Region/Iraq," J. Duhok Univ., vol. 22, no. 1, pp. 1-10, 2019. doi: 10.26682/sjuod.2019.22.1.1.

[25] A. H. A. Al-Ganmi, S. Al-Fayyadh, M. B. H. Abd Ali, A. M. Alotaibi, L. Gholizadeh, and L. Perry, "Medication adherence and predictive factors in patients with cardiovascular disease: A comparison study between Australia and Iraq," Collegian, vol. 26, no. 3, pp. 355-365, 2019. doi: 10.1016/j.colegn.2018.10.002.

[26] H. M. Ahmed, "Quality of Life of Women from Families of Martyred Individuals in the Kurdistan Region of Iraq as a Conflict Area in the Middle East," 2020.

[27] M. Musa Jaber, A. Ghani, M. Khanapi, and N. Suryana Herman, "A Review of Adoption of Telemedicine in 
Middle East Countries: Toward Building Iraqi Telemedicine Framwork.," Sci. Int., vol. 26, no. 5, 2014.

[28] A. Mohammed, M. A. Burhanuddin, A. Alkhazraji, H. Basiron, and D. Tunggal, "IoT Devices and Sensors Management Framework for Mobile E-health Applications," J. Adv. Res. Dyn. Control Syst., vol. 10, pp. $2157-$ 2161, 2018.

[29] A. K. Al-Swidi and M. K. Faaeq, "How robust is the UTAUT theory in explaining the usage intention of egovernment services in an unstable security context?: A study in Iraq," Electron. Gov. an Int. J., vol. 15, no. 1, pp. 37-66, 2019. doi: 10.1504/EG.2019.096580.

[30] M. L. Farnese, B. Barbieri, A. Chirumbolo, and G. Patriotta, "Managing knowledge in organizations: A Nonaka's SECI model operationalization," Front. Psychol., vol. 10, p. 2730, 2019. doi: 10.3389/fpsyg.2019.02730.

[31] D. Weir and K. Hutchings, "Cultural embeddedness and contextual constraints: knowledge sharing in Chinese and Arab cultures," Knowl. Process Manag., vol. 12, no. 2, pp. 89-98, 2005. doi: 10.1002/kpm.222.

[32] Y. I. R. Ayub, O. P. Kogeda, and M. Lall, "Capturing tacit knowledge: A case of traditional doctors in Mozambique," South African J. Inf. Manag., vol. 20, no. 1, pp. 1-8, 2018. doi: 10.4102/sajim.v20i1.880.

[33] W. L. Oberkampf and T. G. Trucano, "Verification and validation benchmarks," Nucl. Eng. Des., vol. 238, no. 3, pp. 716-743, 2008. doi: 10.1016/j.nucengdes.2007.02.032.

[34] E. Cabrera and J. Holt, "An Evaluation of Website Usability for a Cover Crops Resource in the Southern Region.," J. Appl. Commun., vol. 104, no. 1, pp. 1-14, 2020. doi: 10.4148/1051-0834.2305.

[35] P. Acosta-Vargas, L. A. Salvador-Ullauri, and S. Luján-Mora, "A heuristic method to evaluate web accessibility for users with low vision,” IEEE Access, vol. 7, pp. 125634-125648, 2019. doi: 10.1109/ACCESS.2019.2939068.

\section{BIOGRAPHIES OF AUTHORS}
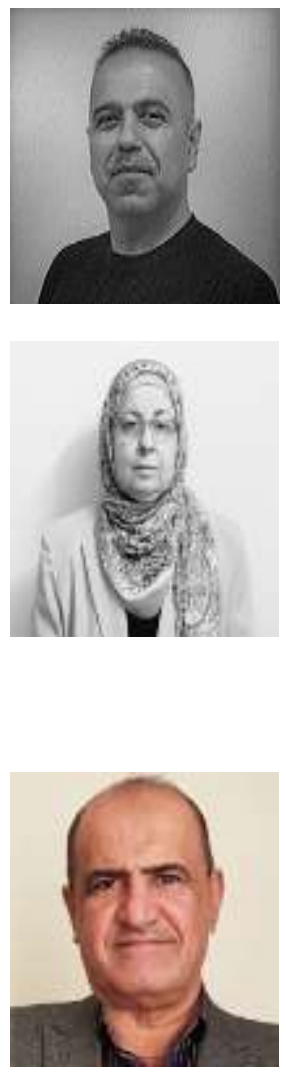

Ahmed S. Aljuboori received a Bachelor of Computer Science degree from the University of Baghdad, Iraq in 2006, and a Master of Computing degree from Manchester Metropolitan University, UK in 2009, and a Ph.D. degree in Data Mining at the University of Salford, UK in 2017. He is a lecturer at the College of Education for Pure Science Ibn-Al-Haitham, University of Baghdad since 2006. His current research interests include the areas of big data, association based on classification and semantic web. Dr. Ahmed Aljuboori is a reviwer in many decent journals such as IEEE open source journal.

Lubab Ahmed Tawfeeq received a Bachelor of Computer Science degree from the University of Baghdad, Iraq in 2003, and a Master of Computing degree from Arab Academy for Science Technology \& Maritime Transport, Egypt in 2013, She is a lecturer at the College of Education for Pure Science Ibn-Al-Haitham, University of Baghdad since 2005, Her current research interests include the areas of image processing and meachine learning. She has published her first paper in 2nd International Conference on Computer Science Innovation \& Technology 2013, Egypt, tilted "Multiple Classifiers For Land Cover Classification \& Change Detection In South Western Iraq", Her second publication was in the Baghdad Science Journal 2019, University of Baghdad, tilted "Reconstruction of Three-Dimensional Object from TwoDimentional Images by Utilizing Distance Regularized Level Algorithm and Mesh Object Generation ".

Khamis A. Al-Karawi received a BSc degree in computer science from Baghdad University, Iraq in 1997. He received an Mc.s in computer science from Pune University, India in 2010. He is received a PhD from Salford University, United Kindom in 2018. Currently he is a lecturer at Diyala university-Iraq. His research intrest is in Automatic speaker recognition, data mining and machine learning. 